\title{
CORRESPONDENCE
}

\section{Tuberculosis mimicry}

\section{To the Editors:}

In a recent issue of the European Respiratory Journal, TAY et al. [1] presented the interesting article on rather rare cases of paragonimiasis in Europe. Taking into consideration tourist activity and growing numbers of oriental restaurants, the probability of this type of infestation in our region is constantly increasing. However, this case is presented from the aspect of differential diagnosis with tuberculosis (TB) and, therefore, requires some comments.

It is underlined that, in Asia, during diagnostic differentiation of infiltrations in the lower zones of lungs, TB, paragonimiasis and melioidosis should be taken into consideration [2]. In TB, changes in the lower areas of lungs without any nodules or cavities in the upper areas are rare. Sometimes infiltrations are only observed in the lower lung zones in lobar pneumonia or bronchopneumonia; however, the radiological appearance of the infiltrations is characteristic and different compared with the presented case [2]. Atypical TB infiltrations in lower areas of the lungs are commonly seen, but only in immunocompromised patients [3].

In the presented case, only a slightly positive tuberculin skin test was noticed, and there was no information regarding the $\mathrm{TB}$ vaccination or comments regarding the positivity of the tuberculin skin test in the population of Singapore.

One of the most important diagnostic investigations in TB is direct examination of sputum and culture for acid-fast bacilli. It is not clear how many sputum samples were collected. According to World Health Organization guidelines for TB control, three sputum examinations are required before implementation of the treatment [4]. Additionally, negative direct sputum examinations and cultures for acid-fast bacilli in patients who expectorate purulent and blood-streaked sputum are infrequent. In this clinical situation, suspicion of diseases other than TB should be considered. Taking all these remarks into consideration, more discerning investigations are required. In our opinion, the probability of $\mathrm{TB}$ in this case was very low.

The following comments concern diagnostic and therapeutic rules. Four months of anti-TB therapy, despite clinical deterioration and progression of radiological changes in patients with negative Mycobacterium smears and cultures, is rather too long. Sometimes, in difficult cases, anti-TB therapy is introduced, but patients are evaluated carefully every month [4].

Therefore, in our opinion, this interesting case should not be presented as tuberculosis mimicry.

\section{E. Radzikowska, M. Chabowski and I. Bestry}

National Tuberculosis and Lung Diseases Research Institute, Warsaw, Poland.

\section{REFERENCES}

1 Tay NSWT, Ong KC, Tan SY, Kaw GJL. Tuberculosis mimicry. Eur Respir J 2005; 26: 554-556.

2 Palmer PES. The imaging of tuberculosis with epidemiological, pathological and clinical correlation. Berlin, Springer, 2002.

3 Zumla A, Grange J. Tuberculosis and co-infection with human immunodeficiency virus. In: Madkour MM, ed. Tuberculosis. Berlin, Springer, 2004; pp. 455-480.

4 Maher D, Chaulet P, Spinaci S, Harries A. Treatment of tuberculosis: guidelines for national programs. WHO/TB/ 97/220. Geneva, WHO, 1997.

DOI: $10.1183 / 09031936.06 .00121205$

From the authors:

We would like to thank E. Radzikowska and coworkers for their comments on our case [1]. The patient was treated for tuberculosis (TB), based on the clinical profile of a patient from a high-prevalence region, with symptoms of chronic cough associated with haemoptysis and chest radiograph infiltrates that did not respond to antibiotic therapy.

Although the chest radiograph features were not typical of TB, it is not impossible for TB to present in this way. With regards to the slightly positive tuberculin skin test, it is known that this test is negative in up to $17 \%$ of patients at the time of diagnosis [2].

Whether two or three sputum specimens for acid-fast bacillus (AFB) smear and/or TB cultures should be the standard in the diagnostic work-up for pulmonary TB is debatable. It would depend on factors unique to each setting, such as the laboratory method used for the AFB smear examination, resources available and the local prevalence of TB.

The experience of the Singapore tuberculosis control unit is that it seems appropriate to treat the majority of patients suspected of having radiological pulmonary tuberculosis with negative sputum smear and culture, while closely monitoring these patients for possible alternative diagnoses [3].

\section{N.S.W.T. Tay}

Tan Tock Seng Hospital, Singapore.

\section{REFERENCES}

1 Tay NSWT, Ong KC, Tan SY, Kaw GJL. Tuberculosis mimicry. Eur Respir J 2005; 26: 554-556.

2 RI Menzies. Tuberculin skin testing. In: Reichman LB, Hershfield ES, eds. Tuberculosis: a comprehensive international approach. New York, Marcel Dekker, 2000; pp. 279-322.

3 Pek WY, Chee CB, Wang YT. Bacteriologically-negative pulmonary tuberculosis: the Singapore tuberculosis control unit experience. Ann Acad Med Singapore 2002; 31: 92-96. 